\title{
Impulse voltage distribution and frequency response of intershield windings
}

Mehdi Bagheri, B. T. Phung, Mohammad Salay Naderi

- Department of Electrical and Electronical Engineering

- Nazarbayev University Library

\begin{abstract}
Impulse voltage distributions in continuous disk windings are nonlinear, due to the small series capacitance of the winding. Consequently, transformer manufacturers often design large power transformers with interleaved windings to increase series capacitance, but in doing so reduce the value of the initial impulse voltage distribution coefficient $\alpha$ given by $\alpha=v\left(C_{g} / C_{s}\right)$, (1) where $C_{g}$ is the shunt capacitance and $C_{s}$ is the series capacitance of the winding. Sometimes fully interleaved windings are employed because they are more reliable, ensuring that the transformer will operate properly under lightning strike. However, fully interleaved windings require longer assembly times, and manual welding is necessary. Manufacturers have therefore been using series combinations of continuous and interleaved windings, known as multiple- $\alpha$ type windings [1]. However, this approach also has disadvantages, e.g., the design and analysis of performance under normal and abnormal transient conditions are complicated, and the impedances of continuous disks differ from those of interleaved disks [2], causing reflection of surge traveling waves at connection points. Transformer designers may therefore have to adjust inter-disk distances and repeat transient simulation studies several times, to find an optimum arrangement.
\end{abstract}

Original language English

Article number

7552374

Pages (from-to)

$32-40$

Number of pages

9

Journal

$\underline{\text { IEEE Electrical Insulation Magazine }}$

Volume

32

Issue number

5

State

Published - Sep 12016

Bagheri, M., Phung, B. T., \& Naderi, M. S. (2016). Impulse voltage distribution and frequency response of intershield windings. IEEE Electrical Insulation Magazine, 32(5), 32-40. [7552374]. DOI: 10.1109/MEI.2016.7552374 